\title{
Lie group analysis, exact solutions and conservation laws of $(3+1)$ dimensional a B-type KP equation
}

\author{
Emrullah Yasar \\ Department of Mathematics, Faculty of Arts and Sciences, Uludag University, Bursa, Turkey
}

Received: 26 July 2016, Accepted: 29 October 2016

Published online: 30 October 2016.

\begin{abstract}
In this study, we considered the (3+1) dimensional a B-type Kademtsev-Petviashvili (KP) equation. Using the Lie group analysis, the symmetry reductions and exact analytic solutions were obtained. Traveling wave solutions were also deduced. Lastly, local conservation laws were constructed by using the multiplier and Ibragimov's nonlocal conservation method.
\end{abstract}

Keywords: Lie symmetry,exact solution, conservation laws, B-type Kadomtsev-Petviashvili equation.

\section{Introduction}

Almost all of physical processes which occurs in the nature are modeled by nonlinear evolution equations (NLEEs). We witness these instances in the all brances of physics, engineerings and applied mathematics. Therefore, there have been intensive studies not only modeling of these equations but also obtaining their solutions. In the open literature we observe some analytical and numerical methods such as inverse scattering transform (IST), Hirota bilinear method, the variational iteration method, the Riccati equation expansion method, the pseudo-spectral method, the sine-cosine method, the tanh-sech method, $G^{\prime} / G$ method, Adomian decomposition method, exponential function method, He's variational principle, Lie symmetry method and many more.

The first studies on the soliton theory starts with the Korteweg-de Vries equation. This equation models the the motion of waves in the shallow-water surfaces. The Kadomtsev-Petviashvili (KP) equation and its extensions such as Boussinesq-Kadomtsev-Petviasvili (BKP) or cylindrical-Kadomtsev-Petviasvili (CKP) types are very intensively investigated in the context of soliton theory. Even though, we observe a plenty of studies for obtaining the soliton solutions (topological or nontopological) of many important $(1+1)$ dimensional integrable NLEEs, there exist relatively small amount of studuies for multi-component systems [1].

In this paper, we will study the following (3+1) dimensional B-type Kadomtsev-Petviashvili partial differential equation (PDE) which describes the processes of interaction of exponentially localized structures [2]

$$
u_{z t}-3 u_{x x} u_{y}-3 u_{x} u_{x y}+3 u_{x x}-u_{x x y}=0 .
$$

We observe a lot of works in the literature for Eq.(1). For instance in [2],the author found the bilinear form, bilinear Bäcklund transformation and Lax pair for Eq. (1) using the binary Bell polynomials approach. Based on Hirota's bilinear form and three-wave method, multi-soliton solutions were also deduced. Darvishi et.al. [1], investigate the some physical importance changes of anti-kink solutions of Eq.(1) by invoking the multiple exp-function method (see, also [3]-[7]). We also observe some very well written works related with variant form of Eq.(1) (see, [8]-[17]). In these works, authors 
investigate mainly the soliton and other exact solutions.

The remainder of this paper is organized as follows. Section 2 is devoted to Lie point symmetry generators of Eq.(1) via Lie group analysis. With the help of Lie group generators, symmetry reductions and invariant solutions were constructed. In Section 3, we get exact analytic solutions by power series method. In Section 4, we obtain traveling wave solutions by using the simplest equation method. We prefer Riccati equation as simplest equation. Section 5 is related with conservation laws of Eq.(1). In order to obtain local conservation laws, we exploit multiplier and new conservation theorem method. In the final section we give some concluding remarks and the main findings of the paper.

\section{Lie group analysis}

In this section, we present the notations and some of the definitions below. For the details see e.g., [19] and [20].The symmetry group of the B-type Kadomtsev-Petviashvili equation (1) will be generated by the vector field of the form

$$
X=\tau(x, y, z, t, u) \frac{\partial}{\partial t}+\xi(x, y, z, t, u) \frac{\partial}{\partial x}+\mu(x, y, z, t, u) \frac{\partial}{\partial y}+\rho(x, y, z, t, u) \frac{\partial}{\partial z}+\eta(x, y, z, t, u) \frac{\partial}{\partial u} .
$$

Applying the fourth order prolongation $p r^{(4)} X$ to (1) results in an overdetermined system of linear PDEs.The corresponding vector fields of (1) are

$$
\begin{aligned}
& X_{1}=\frac{\partial}{\partial t}, X_{2}=\frac{\partial}{\partial x}, X_{3}=\frac{\partial}{\partial y}, X_{4}=\frac{\partial}{\partial z}, \\
& X_{5}=(u-y) \frac{\partial}{\partial u}-3 t \frac{\partial}{\partial t}-x \frac{\partial}{\partial x}, X_{6}=-t \frac{\partial}{\partial t}+z \frac{\partial}{\partial z}, \\
& X_{7}=t \frac{\partial}{\partial t}+y \frac{\partial}{\partial u}+y \frac{\partial}{\partial y} .
\end{aligned}
$$

To obtain the group transformation which is generated by the infinitesimal generators $X_{i}$ for $i=1,2, \ldots 7$, we need to solve the following initial problems ([19] and [21]),

$$
\begin{aligned}
& \frac{d(\bar{x}(\varepsilon))}{d \varepsilon}=\xi(\bar{x}, \bar{y}, \bar{z}, \bar{t}, \bar{u}), \bar{x}(0)=x, \\
& \frac{d(\bar{y}(\varepsilon))}{d \varepsilon}=\mu(\bar{x}, \bar{y}, \bar{z}, \bar{t}, \bar{u}), \bar{y}(0)=y, \\
& \frac{d(\bar{z}(\varepsilon))}{d \varepsilon}=\rho(\bar{x}, \bar{y}, \bar{z}, \bar{t}, \bar{u}), \bar{z}(0)=z, \\
& \frac{d(\bar{t}(\varepsilon))}{d \varepsilon}=\tau(\bar{x}, \bar{y}, \bar{z}, \bar{t}, \bar{u}), \bar{t}(0)=t
\end{aligned}
$$

where $\varepsilon$ is a parameter. So we can obtain the Lie symmetry group

$$
g:(x, y, z, t, u) \rightarrow(\bar{x}, \bar{y}, \bar{z}, \bar{t}, \bar{u}) .
$$

We can build the one-parameter groups $g_{i}$ generated by $X_{i}$ for $i=1,2, \ldots 7$. For instance, the $g_{6}(\varepsilon)$ generated by $X_{6}$ is

$$
g_{6}(\varepsilon):(x, y, z, t, u) \rightarrow\left(x, y, e^{-\varepsilon} z, e^{\varepsilon} t, u\right) .
$$


Transformation (5) corresponds to scaling symmetry. If $u=f(x, y, z, t)$ is a solution of fourth-order Eq.(1), so is the function

$$
g_{6}(\varepsilon) \cdot f(x, y, z, t)=f\left(x, y, e^{-\varepsilon} z, e^{\varepsilon} t\right) .
$$

If taking the following periodic kink-wave solution of Eq. (1) (see, [1]),

$$
u(x, y, z, t)=2 \frac{2 a_{1} \sqrt{\delta_{3}} \sinh \left(\xi_{1}+\ln \sqrt{\delta_{3}}\right)-a_{2} \delta_{1} \sin \left(\xi_{2}\right)}{2 \sqrt{\delta_{3}} \cosh \left(\xi_{1}+\ln \sqrt{\delta_{3}}\right)+\delta_{1} \cos \left(\xi_{2}\right)} .
$$

where

$$
\begin{aligned}
& \xi_{1}=a_{1} x+\frac{a_{1}\left(-3 b_{2} a_{2}^{2}+a_{1}^{2} b_{2}-6 a_{2}\right)}{c_{2}} z \\
& \xi_{2}=a_{2} x+b_{2} y+c_{2} t+\left(\frac{3 a_{1}^{2}-b_{2} a_{2}^{3}+3 a_{1}^{2} b_{2} a_{2}-3 a_{2}^{2}}{c_{2}}\right) z
\end{aligned}
$$

and

$$
\delta_{3}=\frac{1}{4} \frac{\delta_{1}^{2}\left(a_{1}^{2}+b_{2} a_{2}^{3}+a_{1}^{2} b_{2} a_{2}\right)}{a_{1}^{2}} .
$$

one can obtain show that exact solution of Eq. (1) by applying $g_{6}(\varepsilon)$ is as follows

$$
u(x, y, z, t)=2 \frac{2 a_{1} \sqrt{\delta_{3}} \sinh \left(\xi_{1}+\ln \sqrt{\delta_{3}}\right)-a_{2} \delta_{1} \sin \left(\xi_{2}\right)}{2 \sqrt{\delta_{3}} \cosh \left(\xi_{1}+\ln \sqrt{\delta_{3}}\right)+\delta_{1} \cos \left(\xi_{2}\right)}
$$

where

$$
\begin{aligned}
& \xi_{1}=a_{1} x+\frac{a_{1}\left(-3 b_{2} a_{2}^{2}+a_{1}^{2} b_{2}-6 a_{2}\right)}{c_{2}}\left(e^{-\varepsilon} z\right) \\
& \xi_{2}=a_{2} x+b_{2} y+c_{2} e^{\varepsilon} t+\left(\frac{3 a_{1}^{2}-b_{2} a_{2}^{3}+3 a_{1}^{2} b_{2} a_{2}-3 a_{2}^{2}}{c_{2}}\right) e^{-\varepsilon} z
\end{aligned}
$$

Now we obtain some symmetry reductions and invariant solutions for the B-type KP equation based on the vector fields (3).

(1) $X_{1}$, For this case, we obtained the following invariant solution

$$
u(x, y, z, t)=2 c_{2} \tanh \left(\frac{1}{4} \frac{4 c_{2}^{2} x+4 c_{2} c_{4} z+4 c_{1} c_{2}+3 y}{c 2}\right)+c_{5}
$$

where $c_{1}, c_{2}, c_{3}, c_{4}$ and $c_{5}$ are arbitrary constants. This solution is invariant with respect to time translation of the considered equation (1).

(2) $X_{2}$, For this case, we obtained the following invariant solution

$$
u(x, y, z, t)=f(y, z)+g(y, t)
$$

where $f$ and $g$ are arbitrary functions of the indicated arguments.

(3) $X_{4}$, For this case, we obtained the following invariant solution

$$
u(x, y, z, t)=2 c_{2} \tanh \left(\frac{1}{4} \frac{4 c_{2}^{2} x+4 c_{2} c_{4} t+4 c_{1} c_{2}+3 y}{c 2}\right)+c_{5}
$$

where $c_{1}, c_{2}, c_{3}, c_{4}$ and $c_{5}$ are arbitrary constants. 
(4) $X_{5}$, For the generator $X_{5}$, we get

$$
u(x, y, z, t)=\frac{x y+\Phi(f, g, h)}{x}
$$

where $f=y, g=z, h=\frac{t}{x^{3}}$ are the group invariants. Substituting (7) into (1), we get

$$
-27 h^{2} \Phi_{h h} \Phi_{f}-27 h^{2} \Phi_{h} \Phi_{f h}+27 h^{3} \Phi_{h h h f}-63 h \Phi_{h} \Phi_{f}-9 \Phi \Phi_{f}+114 h \Phi_{h f}+6 \Phi_{f}+\Phi_{h g}=0 .
$$

(5) $X_{7}$, In this case, one can obtain

$$
u(x, y, z, t)=y+\Phi(f, g, h)
$$

where $f=x, g=z, h=\frac{t}{y}$ are the group invariants. Substituting (9) into (1), we get

$$
3 h \Phi_{f f} \Phi_{h}+3 h \Phi_{f} \Phi_{f h}+h \Phi_{h f f f}+\Phi_{h g}=0
$$

We can also obtain different type reductions and solutions. First we make use of the symmetry $X=X_{2}+X_{3}+X_{4}$ and reduce the B-type Kadomtsev-Petviashvili equation (1) to a PDE in three independent variables [22].

The symmetry yields the following four invariants:

$$
f=z-y, g=t, h=x-y, \theta=u \text {. }
$$

Treating $\theta$ as the new dependent variable and $f, g$ and $h$ as new independent variables, the B-type Kadomtsev-Petviashvili equation (1) transforms to

$$
\theta_{f g}+\theta_{h h h f}+\theta_{h h h h}+3 \theta_{h} \theta_{f h}+6 \theta_{h} \theta_{h h}+3 \theta_{f} \theta_{h h}+3 \theta_{h h}=0
$$

which is an nonlinear partial differential equation (NLPDE) in three independent variables. We now further reduce (12) using its symmetries. It can be shown that equation (12) has the following seven Lie point symmetries:

$$
\begin{aligned}
& \Upsilon_{1}=\frac{\partial}{\partial f}, \Upsilon_{2}=\frac{\partial}{\partial g}, \Upsilon_{3}=\frac{\partial}{\partial h}, \Upsilon_{4}=\frac{\partial}{\partial \theta}, \Upsilon_{5}=F_{1}(g) \frac{\partial}{\partial \theta} \\
& \Upsilon_{6}=(h-2 f) \frac{\partial}{\partial \theta}+3 g \frac{\partial}{\partial h}, \\
& \Upsilon_{7}=(\theta+2 f) \frac{\partial}{\partial \theta}-f \frac{\partial}{\partial f}-3 g \frac{\partial}{\partial g}-h \frac{\partial}{\partial h} .
\end{aligned}
$$

The symmetry $\Upsilon_{2}+\Upsilon_{3}$ yields the three invariants

$$
r=f, s=g-h, \phi=\theta
$$

which gives a group-invariant solution $\phi=\phi(r, s)$ that satisfies an NLPDE in two independent variables

$$
\phi_{r s}-\phi_{s s s r}+\phi_{s s s s}+3 \phi_{s} \phi_{r s}-6 \phi_{s} \phi_{s s}+3 \phi_{r} \phi_{s s}+3 \phi_{s s}=0
$$

The symmetry algebra of (13) is generated by the vector fields

$$
\Sigma_{1}=\frac{\partial}{\partial r}, \Sigma_{2}=\frac{\partial}{\partial s}, \Sigma_{3}=\frac{\partial}{\partial \phi}, \Sigma_{4}=\left(\phi+\frac{2}{3} s+\frac{10}{3} r\right) \frac{\partial}{\partial \phi}-r \frac{\partial}{\partial r}-s \frac{\partial}{\partial s}
$$


The combination $\alpha \Sigma_{1}+\Sigma_{2}$ of the two symmetries $\Sigma_{1}$ and $\Sigma_{1}$ yields the following invariants

$$
\xi=r-\alpha s, \Psi=\phi
$$

and consequently using these invariants (13) is transformed to the fourth-order nonlinear ordinary differential equation (NLODE)

$$
-\alpha \Psi^{\prime \prime}+\alpha^{3} \Psi^{I V}+\alpha^{4} \Psi^{I V}+6 \alpha^{2} \Psi^{\prime} \Psi^{\prime \prime}+6 \alpha^{3} \Psi^{\prime} \Psi^{\prime \prime}+3 \alpha^{2} \Psi^{\prime \prime}=0
$$

Integration of (14) with respect to $\xi$ two times leads to a second-order variable separable ODE, which can be integrated easily ( taking the constants of integration to be zero ). Then reverting back to the original variables, we can obtain the following solution of the B-type Kadomtsev-Petviashvili equation (1)

$$
u(x, y, z, t)=\frac{(3 \alpha-1) \sin \left(\frac{1}{2} c_{1} \sqrt{\frac{3 \alpha-1}{\alpha^{2}(\alpha+1)}}-\frac{1}{2}(z-y-\alpha(t-x+y)) \sqrt{\frac{3 \alpha-1}{\alpha^{2}(\alpha+1)}}\right)}{\alpha(\alpha+1) \sqrt{\frac{3 \alpha-1}{\alpha^{2}(\alpha+1)}} \cos \left(\frac{1}{2} c_{1} \sqrt{\frac{3 \alpha-1}{\alpha^{2}(\alpha+1)}}-\frac{1}{2}(z-y-\alpha(t-x+y)) \sqrt{\frac{3 \alpha-1}{\alpha^{2}(\alpha+1)}}\right)}+c_{2}
$$

where $c_{1}$ and $c_{2}$ are arbitrary integration constants.

\section{Power series solution of Eq.(1)}

Our aim is to investigate a solution of (14)

$$
\left(\alpha^{3}+\alpha^{2}\right) \Psi^{I V}+(3 \alpha-1) \Psi^{\prime \prime}+\left(6 \alpha+\alpha^{2}\right) \Psi^{\prime} \Psi^{\prime \prime}=0
$$

in a power series of the form ([23])

$$
\Psi=\sum_{n=0}^{\infty} c_{n} \xi^{n}=c_{0}+c_{1} \xi+c_{2} \xi^{2}+c_{3} \xi^{3}+\ldots+c_{n} \xi^{n}+\ldots
$$

Substituting (16) into (15), one can get

$$
\begin{aligned}
& 24\left(\alpha^{3}+\alpha^{2}\right) c_{4}+\left(\alpha^{3}+\alpha^{2}\right)\left[\sum_{n=1}^{\infty}(n+4)(n+3)(n+2)(n+1) c_{n+4} \xi^{n}\right] \\
& +2(3 \alpha-1) c_{2}+(3 \alpha-1)\left[\sum_{n=1}^{\infty}(n+2)(n+1) c_{n+2} \xi^{n}\right]+2\left(6 \alpha+\alpha^{2}\right) c_{1} c_{2} \\
& +\left(6 \alpha+\alpha^{2}\right) \sum_{n=1}^{\infty} \sum_{j=0}^{\infty}(n+1)(n+1-j)(n+2-j) c_{n+1} c_{n+2-j} \xi^{n}=0 .
\end{aligned}
$$

Next, from (17), for the case of $n=0$, one gets

$$
c_{4}=\frac{(1-3 \alpha) c_{2}-\left(6 \alpha+\alpha^{2}\right) c_{1} c_{2}}{12\left(\alpha^{3}+\alpha^{2}\right)}
$$

Generally, for $n \geq 1$, one obtains

$$
\begin{aligned}
c_{n+4} & =\frac{1}{\left(\alpha^{3}+\alpha^{2}\right)(n+4)(n+3)(n+2)(n+1)}\left((1-3 \alpha)(n+2)(n+1) c_{n+2}\right. \\
& \left.-\left(6 \alpha+\alpha^{2}\right) \sum_{j=0}^{\infty}(n+1)(n+1-j)(n+2-j) c_{n+1} c_{n+2-j}\right) .
\end{aligned}
$$


Thus, the power series solution of (16) is as follows

$$
\begin{aligned}
\Psi(\xi) & =c_{0}+c_{1} \xi+c_{2} \xi^{2}+c_{3} \xi^{3}+c_{4} \xi^{4}+\sum_{n=1}^{\infty} c_{n+4} \xi^{n+4}=c_{0}+c_{1} \xi+c_{2} \xi^{2}+c_{3} \xi^{3} \\
& +\frac{(1-3 \alpha) c_{2}-\left(6 \alpha+\alpha^{2}\right) c_{1} c_{2}}{12\left(\alpha^{3}+\alpha^{2}\right)} \xi^{4}+\sum_{n=1}^{\infty} \frac{1}{\left(\alpha^{3}+\alpha^{2}\right)(n+4)(n+3)(n+2)(n+1)}\left((1-3 \alpha)(n+2)(n+1) c_{n+2}\right. \\
& \left.-\left(6 \alpha+\alpha^{2}\right) \sum_{j=0}^{\infty}(n+1)(n+1-j)(n+2-j) c_{n+1} c_{n+2-j}\right) \xi^{n+4}
\end{aligned}
$$

Consequently, the exact power series solution of (1) can be written as follows

$$
\begin{aligned}
u(x, y, z, t) & =c_{0}+c_{1}(z-y-\alpha(t-x+y))+c_{2}(z-y-\alpha(t-x+y))^{2}+c_{3}(z-y-\alpha(t-x+y))^{3} \\
& +c_{4}(z-y-\alpha(t-x+y))^{4}+\sum_{n=1}^{\infty} \frac{1}{\left(\alpha^{3}+\alpha^{2}\right)(n+4)(n+3)(n+2)(n+1)}\left((1-3 \alpha)(n+2)(n+1) c_{n+2}\right. \\
& \left.-\left(6 \alpha+\alpha^{2}\right) \sum_{j=0}^{\infty}(n+1)(n+1-j)(n+2-j) c_{n+1} c_{n+2-j}\right)(z-y-\alpha(t-x+y))^{n+4}
\end{aligned}
$$

where $c_{i}(i=0,1,2,3,4)$ are arbitrary constants, the other coefficients $c_{n}(n \geq 4)$ also can derived.

\section{Traveling wave solutions of Eq.(1) by using simplest equation method}

Now, we intend to derive traveling wave solutions of Eq.(1). For this goal, we use the following general Riccati equation

$$
\varphi^{\prime}=a+b \varphi+c \varphi^{2}
$$

as auxiliary equation where $a, b, c$ are real constants. The general solution of (22) is

$$
\varphi=\frac{\sqrt{4 a c-b^{2}}}{2 b} \frac{C_{1} e^{\frac{\theta}{2} \sqrt{4 a c-b^{2}}}-C_{2} e^{-\frac{\theta}{2} \sqrt{4 a c-b^{2}}}}{C_{1} e^{\frac{\theta}{2} \sqrt{4 a c-b^{2}}}+C_{2} e^{-\frac{\theta}{2} \sqrt{4 a c-b^{2}}}}-\frac{a}{2 b}
$$

where $C_{1}, C_{2}$ are arbitrary constants [23]. By balancing the highest derivative and nonlinear terms in (15), we assume the solution of (1) of the form

$$
f(\theta)=a_{0}+a_{1} \varphi
$$

where $a_{0}, a_{1}$ are constants to be determined. Substituting the ansatz (23) along with (22) into (15), collecting coefficients of monomials of $u$ with the aid of Maple, and then setting each coefficients equal to zero, one gets

$$
a_{0}=a_{0}, a_{1}=\frac{-3\left(\alpha^{3} a^{2}+\alpha^{2} a^{2}+3 \alpha-1\right)}{\alpha c(\alpha+6)}, \alpha=\alpha, a=a, b=\frac{1}{4} \frac{\alpha^{3} a^{2}+\alpha^{2} a^{2}+3 \alpha-1}{\alpha^{2} c(\alpha+1)}, c=c
$$

From the ansatz (23) and making use of Eq. (24), one can get the explicit solution of (1)

$$
u(x, y, z, t)=\frac{-3\left(\alpha^{3} a^{2}+\alpha^{2} a^{2}+3 \alpha-1\right)}{\alpha c(\alpha+6)}\left(\frac{\sqrt{4 a c-b^{2}}}{2 b} \frac{C_{1} e^{\frac{\theta}{2} \sqrt{4 a c-b^{2}}}-C_{2} e^{-\frac{\theta}{2} \sqrt{4 a c-b^{2}}}}{C_{1} e^{\frac{\theta}{2} \sqrt{4 a c-b^{2}}}+C_{2} e^{-\frac{\theta}{2} \sqrt{4 a c-b^{2}}}}-\frac{a}{2 b}\right)+a_{0}
$$

where $b=\frac{1}{4} \frac{\alpha^{3} a^{2}+\alpha^{2} a^{2}+3 \alpha-1}{\alpha^{2} c(\alpha+1)}$ and $\xi=r-\alpha s$. 
In particular, if we set $C_{1}=1$, and $C_{2}=14 a c-b^{2}>0$, one can derive

$$
u(x, y, z, t)=\frac{-3\left(\alpha^{3} a^{2}+\alpha^{2} a^{2}+3 \alpha-1\right)}{\alpha c(\alpha+6)}\left(\frac{\sqrt{4 a c-b^{2}}}{2 b} \tanh \left(\frac{\xi}{2} \sqrt{4 a c-b^{2}}\right)-\frac{a}{2 b}\right)+a_{0} .
$$

\section{Conservation laws of Eq. (1)}

Consider a $k$ th-order system of PDEs of $n$ independent variables $x=\left(x^{1}, x^{2}, \ldots, x^{n}\right)$ and $m$ dependent variables $u=$ $\left(u^{1}, u^{2}, \ldots, u^{m}\right)$, namely

$$
E_{\alpha}\left(x, u, u_{(1)}, \ldots, u_{(k)}\right)=0, \quad \alpha=1, \ldots, m
$$

where $u_{(1)}, u_{(2)}, \ldots, u_{(k)}$ denote the collections of all first, second, $\ldots, k$ th order partial derivatives, i. e., $u_{i}^{\alpha}=D_{i}\left(u^{\alpha}\right)$, $u_{i j}^{\alpha}=D_{j} D_{i}\left(u^{\alpha}\right), \ldots$, respectively, with the total derivative operator with respect to $x^{i}$ is given by

$$
D_{i}=\frac{\partial}{\partial x^{i}}+u_{i}^{\alpha} \frac{\partial}{\partial u^{\alpha}}+u_{i j}^{\alpha} \frac{\partial}{\partial u_{j}^{\alpha}}+\ldots, \quad j=1, \ldots, n
$$

where the summation convertion is used whenever appropriate. With $A$ is the differential function space, the $n$-tuple vector

$$
T=\left(T^{1}, T^{2}, \ldots, T^{n}\right), \quad T^{j} \in A, \quad j=1, \ldots, n,
$$

is a conserved vector of (25) if $T^{i}$ satisfied

$$
\left.D_{i} T^{i}\right|_{(25)}=0
$$

the equation (27) is called a local consevation law of system (25).In the following calculations, we will accept $x^{1}=x, x^{2}=$ $y, x^{3}=z, x^{4}=t$.

\subsection{Multiplier method}

It can be shown that every admitted consevation laws arises from multipliers $Q^{\alpha}\left(x, u, u_{(1)}, \ldots\right)$ such that

$$
Q^{\alpha} E_{\alpha}=D_{i} T^{i}
$$

holds identically [19]. In the multiplier approach for conservation laws, one takes the variational derivative of (28) that is,

$$
\frac{\delta}{\delta u^{\beta}}\left(Q^{\alpha} E_{\alpha}\right)=0
$$

holds for arbitrary functions of $u\left(x^{1}, x^{2}, \ldots, x^{n}\right)$ (see also [24] for the software of the computation of conservation laws).

Here, we use a combination of the multiplier and homotopy approach ([19],[20],[24]), to obtain the multipliers and their corresponding conservation laws for B-type KP equation (1).

We consider the multipliers of the form $Q\left(x, y, z, t, u, u_{x}, u_{y}, u_{z}, u_{t}\right)$ for the equation (1). The determining equation (29) for the multipliers takes the form

$$
\frac{\delta}{\delta u}\left[Q\left(u_{z t}-3 u_{x x} u_{y}-3 u_{x} u_{x y}+3 u_{x x}-u_{x x x y}=0\right)\right]=0
$$


Expanding (30),

$$
Q_{t z}=0, Q_{t u_{x}}=0, Q_{y u_{x}}=0, Q_{z u_{x}}=0, Q_{u_{x} u_{x}}=0, Q_{x}=0, Q_{u}=0, Q_{u_{z}}=0, Q_{u_{t}}=0, Q_{u_{y}}=0
$$

we find that the multiplier $Q$ takes the form

$$
Q=c_{1} u_{x}+F(t, y)+G(y, z)
$$

where $F$ and $G$ are arbitrary functions of indicated arguments. The conserved vector $\left(T^{1}, T^{2}, T^{3}, T^{4}\right)$ of (1) satisfies the divergence relation given by

$$
Q\left[u_{z t}-3 u_{x x} u_{y}-3 u_{x} u_{x y}+3 u_{x x}-u_{x x x y}\right]=D_{x} T^{1}+D_{y} T^{2}+D_{z} T^{3}+D_{t} T^{4}
$$

for all arbitrary functions $u(x, y, z, t)$. If we choose $c_{1}=1, F=0, G=0$, from (31) and (32), we have

$$
\begin{aligned}
& u_{x}\left[u_{z t}-3 u_{x x} u_{y}-3 u_{x} u_{x y}+3 u_{x x}-u_{x x x y}\right]=D_{x}\left[-u u_{x} u_{x y}-2 u_{x}^{2} u_{y}+\frac{3}{2} u_{x}^{2}-\frac{1}{2} u_{y} u_{x x x}\right. \\
& \left.+\frac{1}{2} u_{x y} u_{x x}-\frac{1}{2} u_{x} u_{x x y}+\frac{1}{2} u u_{t z}-\frac{1}{2} u u_{x x x y}\right]+D_{y}\left[u u_{x} u_{x x}+\frac{1}{2} u u_{x x x x}\right]+D_{z}\left[-\frac{1}{2} u u_{t x}\right]+D_{t}\left[\frac{1}{2} u_{z} u_{x}\right] .
\end{aligned}
$$

Thus whenever $u(x, y, z, t)$ is a solution of (1), we have

$$
\begin{aligned}
& D_{x}\left[-u u_{x} u_{x y}-2 u_{x}^{2} u_{y}+\frac{3}{2} u_{x}^{2}-\frac{1}{2} u_{y} u_{x x x}+\frac{1}{2} u_{x y} u_{x x}-\frac{1}{2} u_{x} u_{x x y}+\frac{1}{2} u u_{t z}-\frac{1}{2} u u_{x x x y}\right] \\
& \quad+D_{y}\left[u u_{x} u_{x x}+\frac{1}{2} u u_{x x x x}\right]+D_{z}\left[-\frac{1}{2} u u_{t x}\right]+D_{t}\left[\frac{1}{2} u_{z} u_{x}\right]=0
\end{aligned}
$$

Hence we derive the following conserved vector for (1) from (33):

$$
\begin{aligned}
T_{x}^{1}= & -u u_{x} u_{x y}-2 u_{x}^{2} u_{y}+\frac{3}{2} u_{x}^{2}-\frac{1}{2} u_{y} u_{x x x}+\frac{1}{2} u_{x y} u_{x x} \\
& -\frac{1}{2} u_{x} u_{x x y}+\frac{1}{2} u u_{t z}-\frac{1}{2} u u_{x x x y}, \\
T_{y}^{1}= & u u_{x} u_{x x}+\frac{1}{2} u u_{x x x x}, \\
T_{z}^{1}= & -\frac{1}{2} u u_{t x}, T_{t}^{1}=\frac{1}{2} u_{z} u_{x},
\end{aligned}
$$

Now, we take $G$ as arbitrary function, $c_{1}=0$, and $F=0$ then the following conserved vectors are deduced:

$$
T_{x}^{2}=-3 u_{x} u_{y} G(y, z)+3 u_{x} G(y, z), T_{y}^{2}=0, T_{z}^{2}=0, T_{t}^{2}=u_{z} G(y, z)
$$

On the other hand, if we choose $c_{1}=0$, and $G=0$ then we yield the the following conserved vectors:

$$
T_{x}^{3}=-3 u_{x} u_{y} F(t, y)+3 u_{x} F(t, y), T_{y}^{3}=0, T_{z}^{3}=-u F_{t}(t, y), T_{t}^{3}=u_{z} F(t, y) .
$$




\subsection{New conservation theorem method}

It is shown in [25] (see also,[27]-[29]) that, the system and its adjoint equation

$$
\begin{aligned}
F\left(x, u, u_{(1)}, u_{(2)}, \ldots u_{(s)}\right) & =0 \\
F^{*}\left(x, u, v, u_{(1)}, v_{(1)}, u_{(2)}, v_{(2)}, \ldots u_{(s)}, v_{(s)}\right) & =0
\end{aligned}
$$

has a formal Lagrangian, namely

$$
L=v F\left(x, y, z, t, u, u_{(1)}, u_{(2)}, \ldots u_{(s)}\right)
$$

where adjoint equation (37) is defined as

$$
F^{*}\left(x, u, v, u_{(1)}, v_{(1)}, u_{(2)}, v_{(2)}, \ldots u_{(s)}, v_{(s)}\right)=\frac{\delta L}{\delta u}
$$

where $\frac{\delta}{\delta u}$ is variational derivative

$$
\frac{\delta}{\delta u}=\frac{\partial}{\partial u}+\sum_{s=1}^{\infty}(-1)^{s} D_{i_{1}} \ldots D i_{s} \frac{\partial}{\partial u_{i_{1} \cdots i_{s}}} .
$$

The equation (35) is said to be nonlinearly self-adjoint if for some arbitrary function $\phi(t, x, y, z) \neq 0$, we have

$$
\left.F^{*}\right|_{v=\phi}=\lambda\left(x, u, u_{(1)}, \ldots\right) F,
$$

where $\lambda$ is an indeterminate variable coefficient [26] (see also ([21] and [23]).

In [25], Ibragimov proved that, every Lie point, Lie-Bäcklund and non-local symmetry of Eq.(35) provides a conservation law for Eq. (35) and the adjoint equation (36). Then the elements of conservation vector $T^{1}, T^{2}, T^{3}, T^{4}, \ldots$ are given by

$$
\begin{aligned}
T^{i} & =\xi^{i} L+W^{\alpha}\left[\frac{\partial L}{\partial u_{i}}-D_{j}\left(\frac{\partial L}{\partial u_{i j}}\right)+D_{j} D_{k}\left(\frac{\partial L}{\partial u_{i j k}}\right)-D_{j} D_{k} D_{m}\left(\frac{\partial L}{\partial u_{i j k m}}\right)+\ldots\right] \\
& +D_{j}\left(W^{\alpha}\right)\left[\frac{\partial L}{\partial u_{i j}}-D_{k}\left(\frac{\partial L}{\partial u_{i j k}}\right)+D_{k} D_{m}\left(\frac{\partial L}{\partial u_{i j k m}}\right)+\ldots\right] \\
& +D_{j} D_{k}\left(W^{\alpha}\right)\left[\frac{\partial L}{\partial u_{i j k}}-D_{m}\left(\frac{\partial L}{\partial u_{i j k m}}\right)+\ldots\right]+D_{j} D_{k} D_{m}\left(W^{\alpha}\right)\left[\left(\frac{\partial L}{\partial u_{i j k m}}\right)+\ldots\right]
\end{aligned}
$$

First, we will discuss nonlinear self-adjointness of Eq.(1).

For (1), the adjoint equation has the form

$$
F^{*}=-6 u_{x y} v_{x}+\left(-3 u_{y}+3\right) v_{x x}-3 u_{x} v_{x y}+v_{z t}-v_{x x y},
$$

and the formal Lagrangian has the following form

$$
L=v\left(u_{z t}-3 u_{x x} u_{y}-3 u_{x} u_{x y}+3 u_{x x}-u_{x x x y}\right)
$$

If we substitute $u$ instead of $v$ in Eq. (33), one can find that Eq. (1) is not recovered. Therefore, we can say Eq. (1) is not self adjoint. Next,we look for an explicit form of $\phi(x, y, z, t, u) \neq 0$ for Eq. (1) that holds Eq. (41).

$$
-6 u_{x y} v_{x}+\left(-3 u_{y}+3\right) v_{x x}-3 u_{x} v_{x y}+v_{z t}-v_{x x y}=\lambda\left(u_{z t}-3 u_{x x} u_{y}-3 u_{x} u_{x y}+3 u_{x x}-u_{x x x y}\right)
$$


If we plugging them $v_{x}, v_{x x}, v_{y x}, v_{z t}$ and $v_{y x x x}$ into (43), one can arrive first from the coefficient of $u_{z t}$

$$
\lambda=\phi_{u}
$$

Note that the other coefficients of $u$ and all of the derivative yields

$$
\begin{aligned}
\phi_{u u} & =0, \\
-9 \phi_{u}-6 \phi_{x u u} & =-3 \phi_{u u}, \\
-\phi_{u} & =-\phi_{u u}, \\
-3 \phi_{x y u}+3 \phi_{u} & =3 \phi_{u u}, \\
3 \phi_{x x}+\phi_{z t}-\phi_{x x x y} & =0, \\
-6 \phi_{u u}-3 \phi_{x u u u} & =0, \\
-6 \phi_{x}-3 \phi_{x x u} & =0 \\
3 \phi_{u u}-3 \phi_{y u}-3 \phi_{x y u u} & =0 \\
\phi_{z u} & =0 \\
\phi_{y u} & =0 \\
\phi_{t u} & =0 \\
\phi_{x x x u} & =0 .
\end{aligned}
$$

Solve them, one can get the solution

$$
\phi(x, y, z, t)=F_{1}(y, t)+F_{2}(y, z)
$$

where $F_{1}$ and $F_{2}$ are arbitrary functions of arguments.

Therefore, the B-type Kadomtsev-Petviashvili equation is nonlinearly self-adjoint with the substitution $v=\phi$ and $\phi$ is given by (45).

Now, we will study the conservation laws by using the adjoint equation and symmetries of (45). For (45), the adjoint equation has the form Eq. (41) and the Lagrangian in the symmetrized form is (42).

Let us consider the vector field (2) corresponding to conserved vector.The operator $X$ yields the conservation law

$$
D_{t}\left(T^{t}\right)+D_{x}\left(T^{x}\right)+D_{y}\left(T^{y}\right)+D_{z}\left(T^{z}\right)_{\mid(1)}=0
$$

where the conserved vector $T=\left(T^{t}, T^{x}, T^{y}, T^{z}\right)$ is given by (40) and has the components

$$
\begin{aligned}
T^{t} & =\xi^{t} L+W\left[-D_{z}(v)\right]+D_{z}(W)[v], \\
T^{x} & =\xi^{x} L+W\left[-3 v u_{x y}-D_{x}\left(-3 v u_{y}+3 v\right)-D_{x}^{2} D_{y}(-v)-D_{y}\left(-3 v u_{x}\right)-D_{y} D_{x}^{2}(-v)\right] \\
& +D_{x}(W)\left[\left(-3 v u_{y}+3 v\right)+D_{x} D_{y}(-v)\right]+D_{y}(W)\left[-3 v u_{x}+D_{x}^{2}(-v)\right] \\
& +D_{x}^{2}(W)\left[-D_{y}(-v)\right]+D_{y} D_{x}\left[-D_{x}(-v)\right]+D_{y} D_{x}^{2}(W)(-v), \\
T^{y} & =\xi^{y} L+W\left[-D_{x}\left(-3 v u_{x}\right)-D_{x}^{3}(-v)\right]+D_{x}(W)\left[\left(-3 v u_{x}\right)+D_{x}^{2}(-v)\right] \\
& +D_{x}^{2}(W)\left[-D_{x}(-v)\right]+D_{x}^{3}(W)(-v), \\
T^{z} & =\xi^{z} L+W\left[-D_{t}(v)\right]+D_{t}(W)[v] .
\end{aligned}
$$


Therefore, (47)-(50) define the corresponding components of a non-local conservation law for the system of (1) and (41) corresponding to any operator $X$ admitted by (1). Now, let us make calculations for the operator $X_{5}=(u-y) \frac{\partial}{\partial u}-3 t \frac{\partial}{\partial t}-x \frac{\partial}{\partial x}$ in detail.

For this operator, one can get $W=u-y+3 t u_{t}+x u_{x}$, we can get the conservation vector of Eq. (1)

$$
\begin{aligned}
T^{t} & =-3 t v\left(u_{z t}-3 u_{x x} u_{y}-3 u_{x} u_{x y}+3 u_{x x}-u_{x x x y}\right)+\left(u-y+3 t u_{t}+x u_{x}\right)\left[-D_{z}(v)\right]+D_{z}\left(u-y+3 t u_{t}+x u_{x}\right)[v], \\
T^{x} & =-x v\left(u_{z t}-3 u_{x x} u_{y}-3 u_{x} u_{x y}+3 u_{x x}-u_{x x x y}\right)+\left(u-y+3 t u_{t}+x u_{x}\right)\left[-3 v u_{x y}\right. \\
& \left.\left.-D_{x}\left(-3 v u_{y}+3 v\right)\right]-D_{x}^{2} D_{y}(-v)-D_{y}\left(-3 v u_{x}\right)-D_{y} D_{x}^{2}(-v)\right] \\
& +D_{x}\left(u-y+3 t u_{t}+x u_{x}\right)\left[\left(-3 v u_{y}+3 v\right)+D_{x} D_{y}(-v)\right]+D_{y}\left(u-y+3 t u_{t}+x u_{x}\right)\left[-3 v u_{x}+D_{x}^{2}(-v)\right] \\
& +D_{x}^{2}\left(u-y+3 t u_{t}+x u_{x}\right)\left[-D_{y}(-v)\right]+D_{y} D_{x}\left[-D_{x}(-v)\right]+D_{y} D_{x}^{2}\left(u-y+3 t u_{t}+x u_{x}\right)(-v), \\
T^{y} & =\left(u-y+3 t u_{t}+x u_{x}\right)\left[-D_{x}\left(-3 v u_{x}\right)-D_{x}^{3}(-v)\right]+D_{x}\left(u-y+3 t u_{t}+x u_{x}\right)\left[\left(-3 v u_{x}\right)+D_{x}^{2}(-v)\right] \\
& +D_{x}^{2}\left(u-y+3 t u_{t}+x u_{x}\right)\left[-D_{x}(-v)\right]+D_{x}^{3}\left(u-y+3 t u_{t}+x u_{x}\right)(-v), \\
T^{z} & =\left(u-y+3 t u_{t}+x u_{x}\right)\left[-D_{t}(v)\right]+D_{t}\left(u-y+3 t u_{t}+x u_{x}\right)[v] .
\end{aligned}
$$

Substituting the solutions (45) of adjoint equation Eq.(1), one can get infinite number of conservation laws of Eq.(1).

\section{Conclusions}

In this paper, we investigated exact solutions and conservation laws of (3+1) dimensional a B-type Kadomtsev Petviashvili equation (1). The invariant solutions, exact anaytic power series solutions and traveling wave solutions were constructed. The obtained solutions can be utilized a numerical benchmark in the studies of numerical works for Eq.(1). In addition, local and independent conservation laws were obtained by the two distinct methods. The obtained conservation laws can be utilized in the constructing of numerical schemas, stability analysis of solutions and symmetry reductions for getting the new type exact solutions.

\section{Competing interests}

The authors declare that they have no competing interests.

\section{Authors' contributions}

All authors have contributed to all parts of the article. All authors read and approved the final manuscript.

\section{References}

[1] M. T. Darvishi, M. Najafi, S. Arbabi, L. Kavitha, Exact propagating multi-anti-kink soliton solutions of a (3+ 1)-dimensional B-type Kadomtsev-Petviashvili equation, Nonlinear Dynamics, 83(3) (2016), 1453-1462

[2] L. Na, Bäcklund transformation and multi-soliton solutions for the (3+1)-dimensional BKP equation with Bell polynomials and symbolic computation, Nonlinear Dynamics, 82 (2015), 311-318.

[3] W. X. Ma, E.G. Fan, Linear superposition principle applying to Hirota bilinear equations, Comput. Math. Appl, 61 (2011), $950-959$

[4] W. X. Ma, Z. Zhu, Solving the (3+ 1)-dimensional generalized KP and BKP equations by the multiple exp-function algorithm, Appl. Math. Comput, 218 (2012), 11871-11879 
[5] G.Q. Xu, X.Z. Huang, New variable separation solutions for two nonlinear evolution equations in higher dimensions, Chin. Phys. Lett., 30 (2013),130202

[6] E. T. Bell, Exponential polynomials, Ann. Math., 35 (1934), 258-277

[7] C. Gilson, F. Lambert, J. Nimmo, R. Willox, On the combinatorics of the Hirota D-operators, Proc. R. Soc. Lond. Ser. A, 452 , (1996), 223-234

[8] A. Biswas,1-Soliton solution of the generalized Camassa-Holm Kadomtsev-Petviashvili equation, Communications in Nonlinear Science and Numerical Simulation, 14 (6), 2524-2527. (2009).

[9] A. Biswas, A. Ranasinghe, 1-Soliton solution of Kadomtsev-Petviashvili equation with power law nonlinearity, Applied Mathematics and Computation, 214 (2), 645-647. (2009).

[10] A. Biswas, A. Ranasinghe,Topological 1-Soliton solution of Kadomtsev-Petviashvili equation with power law nonlinearity, Applied Mathematics and Computation, 217(4), 1771-1773. (2010).

[11] A.J.M.Jawad, M Petkovic, A. Biswas, Soliton solutions for nonlinear Calogero-Degasperis and Potential Kadomtsev-Petviashvili equation, Computers and Mathematics with Applications,62 (6), 2621-2628. (2011).

[12] H.Triki,B. J. M. Sturdevant, T. Hayat, Omar M. Aldossary, Anjan Biswas, Shock wave solutions of the variants of KadomtsevPetviashvili equation, Canadian Journal of Physics, Volume 89, Number 9, 979-984. (2011).

[13] G. Ebadi, N. Yousefzadeh, H. Triki , A. Biswas, Exact solutions of the (2+1) dimensional Camassa Holm Kadomtsev-Petviashvili equation,Nonlinear Analysis: Modelling and Control, 17 (3), 280-296. (2012).

[14] A. H. Bhrawy, M. A. Abdelkawy, Sachin Kumar, Anjan Biswas, Solitons and other solutions to Kadomtsev-Petviashvili equation of B-type, Romanian Journal of Physics, 58 (7-8), 729-748. (2013).

[15] G. Ebadi, N. Y. Fard, A. H. Bhrawy, S Kumar, H Triki, A Yildirim, Anjan Biswas, Solitons and other solutions to the (3+1) dimensional extended Kadomtsev-Petviashvili equation with power law nonlinearity, Romanian Reports in Physics, 65 (1), $27-62$. (2013).

[16] S. Kumar, E. Zerrad, A Yildirim, A. Biswas.The Kadomtsev-Petviashvili equation-Burgers equation with power law nonlinearity in dust plasmas ,Proceedings of the Romanian Academy, Series A, 14(3), 204-210. (2013).

[17] N. Y. Fard, M R. Foroutan, M Eslami, M Mirzazadeh, A Biswas, Solitary waves and other solutions to Kadomtsev-Petviashvili equation with spatio-temporal dispersion, Romanian Journal of Physics, 60 ( 9-10), 1337-1360. (2015).

[18] A.J. M. Jawad, M Mirzazadeh, A Biswas, Dynamics of shallow water waves with Gardner Kadomtsev-Petviashvili equation, Discrete and Continuous Dynamical Systems, Series S, 8 ( 6), 1155-1164. (2015).

[19] P.J. Olver, Applications of Lie groups to differential equations (Vol. 107), Springer Science \& Business Media, 2000.

[20] G. W. Bluman, S. Kumei, Symmetries and Differential Equations, Springer New York, 1989.

[21] G. W. Wang, X.Q. Liu, Y.Y. Zhang, Symmetry reduction, exact solutions and conservation laws of a new fifth-order nonlinear integrable equation. Communications in Nonlinear Science and Numerical Simulation,18(9) (2013), 2313-2320.

[22] A.R. Adem, C.M. Khalique, A. Biswas, Solutions of Kadomtsev-Petviashvili equation with power law nonlinearity in $1+3$ dimensions. Mathematical Methods in the Applied Sciences, 34(5) (2011), 532-543.

[23] G. Wang, K. Fakhar, Lie symmetry analysis, nonlinear self-adjointness and conservation laws to an extended (2+1)-dimensional Zakharov-Kuznetsov-Burgers equation, Computers \& Fluids, 119,(2015),143-148

[24] A. Cheviakov, GeM software package for computation of symmetries and conservation laws of differential equations. Comp. Phys. Comm. 176 (2007), 48-61

[25] N.H. Ibragimov, A new conservation theorem, J Math Anal Appl, 333 (2007), 311-28.

[26] N.H. Ibragimov, Nonlinear self-adjointness and conservation laws, J Phys A: Math Theor, 44 (2011) 432002.

[27] E. Yaşar, T. Özer, Conservation laws for one-layer shallow water wave systems, Nonlinear Analysis: Real World Applications, 11(2) (2010), 838-848.

[28] E. Yaşar, T. Özer, Invariant solutions and conservation laws to nonconservative FP equation, Computers \& Mathematics with Applications, 59(9) (2010), 3203-3210.

[29] E. Yaşar, T. Özer, On symmetries, conservation laws and invariant solutions of the foam-drainage equation, International Journal of Non-Linear Mechanics, 46(2) (2011), 357-362. 